\title{
Methods for Null Control and Their Effects on the Radiation Pattern
}

\author{
HANS STEYSKAL, MEMBER, IEEE, ROBERT A. SHORE, AND RANDY L. HAUPT, STUDENT MEMBER, IEEE
}

\begin{abstract}
Several methods for synthesis of array antenna patterns with prescribed nulls are reviewed. Methods based on full amplitude/phase control at each array element and methods with a restricted number of degrees of freedom are compared, with attention focused on the characteristic features of the resultant patterns. These features are largely independent of any algorithm for achieving the nulls, and therefore they also provide a perspective on the performance of adaptive antenna systems, which employ these various control architectures.
\end{abstract}

\section{INTRODUCTION}

S YNTHESIS TECHNIQUES which provide control of the nulls of the antenna pattern are of interest for two different, but related reasons. First, they represent generalizations of classical pattern synthesis techniques, which deal with the main beam shape and the sidelobe envelope, but neglect the detailed sidelobe structure. Due to the increasing pollution of the electromagnetic environment, synthesis methods, which allow placing one or more nulls in the pattern at specified directions, are becoming important.

The second reason is that they provide insight into adaptive antenna systems [1]. Discussion of these systems generally emphasizes the choice, implementation and performance of an adaptive algorithm. Equally important however, are such fundamental questions as: what is the optimal control architecture for the system, what is the minimum number of degrees of freedom required, how severe is the pattern deterioration, what is the attainable null depth and what are the ultimate limitations? These questions, which are independent of any particular algorithm, can be addressed by focusing attention on direct pattern null synthesis, since the final state of any adapted system is a pattern with nulls in the directions of the jammers. In this approach one can readily investigate the characteristics of such null-constrained patterns, when synthesized with various forms of control of the array excitation.

Pattern control in an array antenna can be achieved in various ways. Some common configurations are shown in Fig. 1. The most general but also most costly alternative clearly is control of both amplitude and phase of each array element. A second alternative is through perturbation of element phase only, which is attractive since in a phased array the required controls are available at no extra cost. A third method is to incorporate controls only at a select subset of elements or at the subarray level, which is tempting because it reduces the

Manuscript received April 3, 1985; revised August 6, 1985.

The authors are with the Electromagnetic Sciences Division, Rome Air Development Center, Hanscom AFB, MA 01731.

IEEE Log Number 8407023.

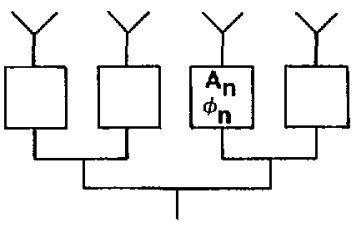

AMPLITUDE/PHASE

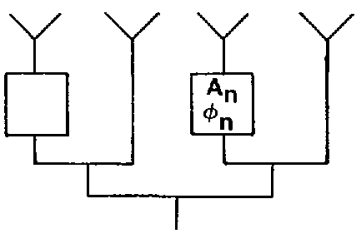

SELECT ELEMENTS

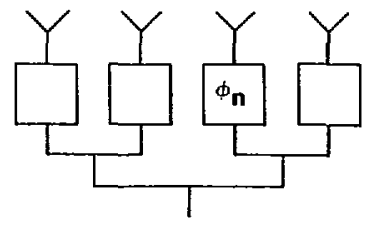

PHASE-ONLY

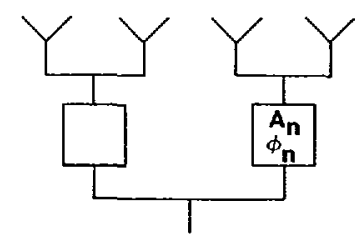

SUBARRAY WEIGHTING
Fig. 1. Typical control configurations.

number of electronic controls. We have analyzed these approaches, the details of which are given in [2]-[7], and we present here a summary of our results only.

A linear array of $2 N+1$ isotropic equispaced elements is considered, which has the pattern

$$
f(u)=\sum_{-N}^{N} a_{n} e^{j n k d u}
$$

where $a_{n}, k, d, u$ denote the element excitation, the wavenumber, the interelement spacing and the sine of the angle $\theta$ from broadside, respectively. The method for pattern null synthesis starts from a given original pattern $f_{0}(u)$, with desired main beam and sidelobe envelope, corresponding to given original element coefficients $\left\{a_{0 n}\right\}$. These coefficients are then perturbed such that the perturbed pattern has nulls at the desired directions. We limit ourselves here to the important special case of real and symmetric coefficients $a_{0 n}$ and hence to real symmetric patterns $f_{0}$.

\section{Pattern Nulling with Full Amplitude/Phase Control}

For the case with full amplitude and phase control we define the desired pattern $f(u)$ mathematically by the criterion

$$
\begin{gathered}
f\left(u_{m}\right)=0, \quad m=1,2, \cdots, M \\
\sum_{n} c_{n}\left|a_{n}-a_{0 n}\right|^{2}=\text { minimum. }
\end{gathered}
$$

Equation (2a) ensures that the synthesized pattern has nulls at the desired directions $u_{m}$, and (2b) ensures that the array excitation is only minimally perturbed. The real positive, 
symmetric weight coefficients $c_{n}$ add flexibility to the criterion.

The solution for the perturbed element coefficients is [2]

$$
a_{n}=a_{0 n}-\sum_{m=1}^{M} \gamma_{m}\left(1 / c_{n}\right) e^{-j n k d u_{m}}
$$

where the beam coefficients $\gamma_{m}$ are determined from an $M$ th order system of linear equations. Hence the perturbed pattern is

$$
f(u)=f_{0}(u)-\sum_{m=1}^{M} \gamma_{m} \sum_{n}\left(1 / c_{n}\right) e^{j n k d\left(u-u_{m}\right)}
$$

Note that in (4) each term in the sum over $m$ represents one cancellation beam with amplitude coefficient $\gamma_{m}$ and the direction $u_{m}$ of the desired null. The null synthesis problem thus has only the dimensionality $M$, rather than the dimensionality $2 N+1$ of element space. This represents a significant simplification of the problem.

The weight coefficients $c_{n}$ determine the shape of the cancellation beams; $c_{n}=1$ leads to beams that are sincfunctions, whereas setting $c_{n}=1 / a_{0 n}$ leads to beams which are replicas of the original pattern $f_{0}$. In the former case the perturbed pattern $f$ can be shown to be the constrained least mean square approximation to $f_{0}$, over one period of the pattern. In the latter case the interpretation is not so simple.

Two examples of Chebyshev patterns, each with four imposed nulls, are shown in Fig. 2. The nulls are placed close together so as to create a sector with very low sidelobes. As seen, the original sidelobe levels have been strongly reduced within a sector $\Delta u=0.06$.

\section{Wide-Band Nulling versus Number of Degrees of}

\section{FREEDOM}

We digress here briefly, before proceeding with phase-only pattern control, to comment on the sidelobe suppression obtained with several closely spaced pattern nulls. This is of importance since it relates to the number of adaptive loops and therefore to the complexity and cost, required by an adaptive antenna system.

An array with a conventional feed network has a frequency dependent antenna pattern such that a point source at a fixed direction $u_{j}$ and with relative frequency bandwidth $B$ (i.e., total bandwidth/center frequency) will appear to be smeared out over an angular pattern sector

$$
\Delta u=B \cdot u_{j}
$$

which is centered at $u=u_{j}$. One way of achieving an approximate wide-band pattern null is therefore to impose a sufficient number of equidistant, discrete nulls over the corresponding pattern sector $\Delta u$.

The question then arises as to the relation between the parameters involved, i.e., the number $M$ of imposed nulls, the sector width $\Delta u$, the sidelobe cancellation $C$ (relative to the original level) and the dependence on the original pattern and the desired null direction $u_{j}$. Although we have no analytic answer, a numerical study [3] has shown that to first order, the

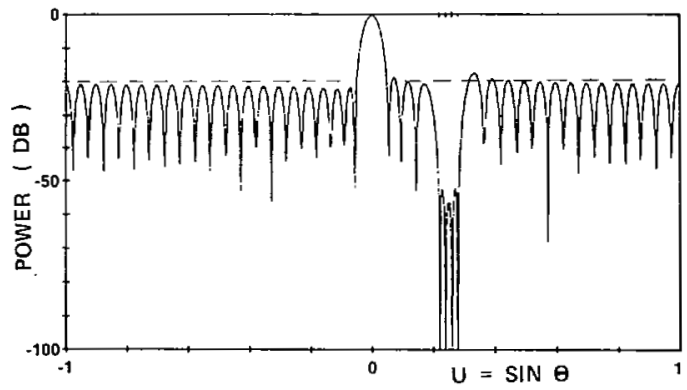

(a)

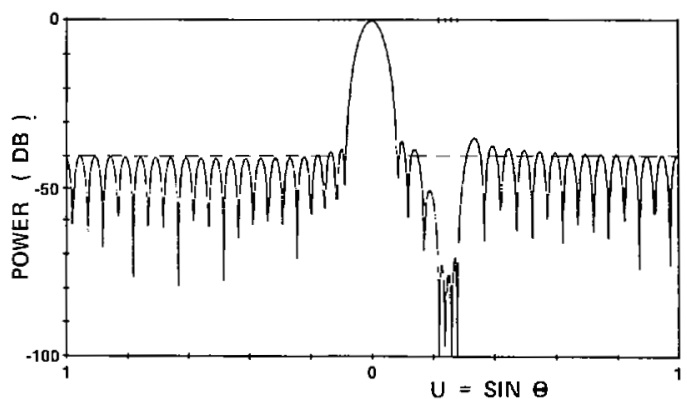

(b)

Fig. 2. (a) Perturbed $20 \mathrm{~dB}$ Chebyshev pattern with four nulls imposed at $u$ $=0.22,0.24,0.26,0.28$. Sidelobe cancellation is $32 \mathrm{~dB} .2 N+1=41, d$ $=\lambda / 2, c_{n}=1$. (b) Perturbed $40 \mathrm{~dB}$ Chebyshev pattern with nulls imposed as in Fig. 2(a). Sidelobe cancellation is $30 \mathrm{~dB}$.

sidelobe cancellation is independent of the actual pattern type and is determined by only two parameters: the number of nulls $M$ and the number of sidelobes $\nu$ to be cancelled. The results are summarized in Fig. 3, which graphs the cancellation $C$ as a function of $M$ and $\nu$. The latter parameter translates into $B$ and $u_{j}$ via the relation $\nu=\Delta u(\lambda / l)=B u_{j} l / \lambda$, since normally for an antenna of length $l$ the sidelobe width is $\lambda / l$.

The patterns in Fig. 2 show 32 and $30 \mathrm{~dB}$ pattern cancellation, whereas for the same case, i.e., $\nu=1, M=4$, a $34 \mathrm{~dB}$ cancellation is obtained from Fig. 3 . This demonstrates the applicability of the graph.

The curves in Fig. 3 are also useful in that they allow a convenient estimate for the number of nulls required to produce the desired sidelobe cancellation. This number is indicative of how many degrees of freedom a conventional array with full amplitude/phase control must allocate to attain a specific nulling performance.

\section{Pattern Nulling with Control of Phase Only}

In the case where only phase perturbations of the original excitation are allowed, the criterion (2) is modified to

$$
\left\{\begin{array}{l}
f\left(u_{m}\right)=0, \quad m=1,2, \cdots, M \\
\sum_{n} c_{n} \mid a_{0 n} e^{j \phi_{n}-\left.a_{0 n}\right|^{2}=\text { minimum }}
\end{array}\right.
$$

where $\left\{\phi_{n}\right\}$ are the desired phase perturbations.

Equation (6) represents a nonlinear problem which does not necessarily have a solution. However, when a solution exists, the phase perturbations can be written in the form [8], [4]

$$
\phi_{n}=\text { phase }\left[a_{0 n}-\sum_{m} \gamma_{m} \frac{1}{c_{n}} e^{-j n k d u} m\right]
$$




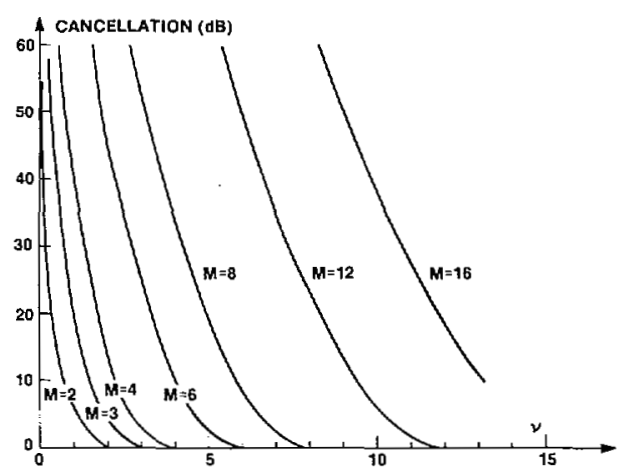

Fig. 3. Sidelobe cancellation versus number of equispaced pattern nulls $M$ and desired number of cancelled sidelobes $\nu$.

which in structure is remarkably similar to (3). Although the unknown beam coefficients $\gamma_{\mathrm{m}}$ are different from those of (3), the "beamspace" representation (7) shows that again the problem is of dimensionality $M$ rather than $2 N+1$. In addition it can be shown [5] that the phase perturbations are odd symmetric $\phi_{-n}=-\phi_{n}$, and hence the coefficients $\gamma_{m}$ are real.

When discussing the patterns resulting from (6) it is proper to distinguish the case where the phase perturbations are small and the case where they have arbitrarily large values.

\section{A. Small Phase Perturbations}

Placing a relatively small number of nulls $(M \ll 2 N+1)$ in a region of low sidelobes constitutes a relatively modest pattern perturbation and therefore it is reasonable to expect that the associated phase perturbations are also small, $\left|\phi_{n}\right| \ll$ 1. In this case, a two-term Taylor expansion of the phase term in (6b) leads to

$$
\begin{aligned}
& f\left(u_{m}\right) \approx f_{0}\left(u_{m}\right)+j \Sigma a_{0 n} \phi_{n} e^{j n k d u_{m}}=0 \\
& \sum_{n} c_{n}\left(a_{0 n} \phi_{n}\right)^{2}=\text { minimum }
\end{aligned}
$$

which constitutes a linear problem with an analytic solution [2], [6].

The resultant pattern can again be viewed as the original pattern on which $M$ cancellation beams have been superimposed. This time, however, each cancellation beam consists of a beam pair, where the two members have opposite sign and are directed at $u_{m}$ and $-u_{m}$, respectively. The coefficients $c_{n}$ determine the shape of each member in the same way as before: choosing $c_{n}=1$ leads to a pair of sinc beams, whereas $c_{n}=1 / a_{0 n}$ leads to a pair of original beam replicas.

Two examples of patterns with one imposed null at $\theta=$ $15.23^{\circ}$ and their corresponding cancellation beams are shown in Figs. 4 and 5, for $c_{n}=1$ and $c_{n}=1 / a_{0 n}$. Apparently, the choice of the weighting coefficients $c_{n}$ has no dramatic effects and in both cases a deep null $(<-100 \mathrm{~dB})$ is achieved. Note also the corresponding sidelobe level increase at $\theta=$ $-15.23^{\circ}$ caused by the second member of the cancellation beam pair. Further examples with multiple nulls may be found in [2], [6].

The null synthesis method based on the small phase perturbation approximation is satisfactory for most situations.

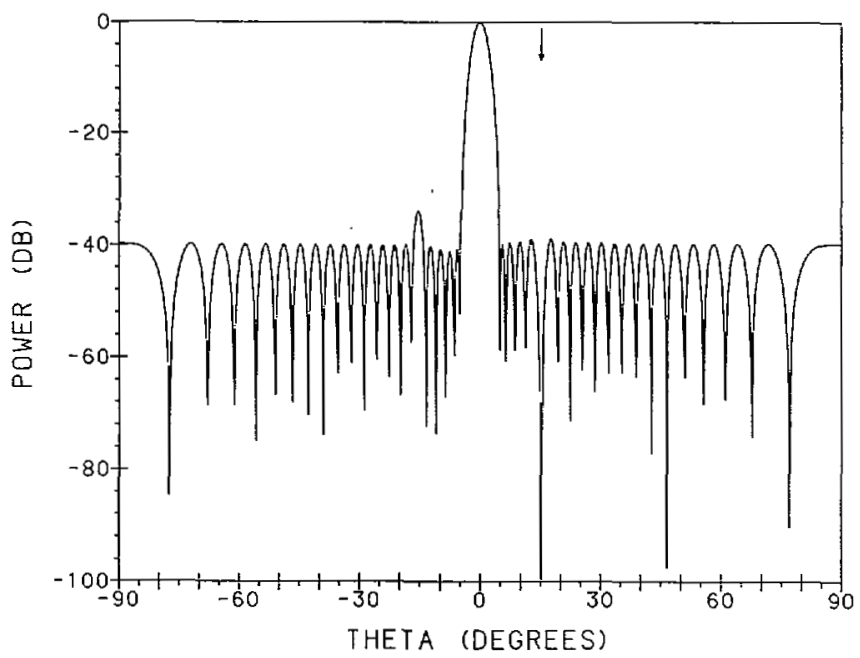

(a)

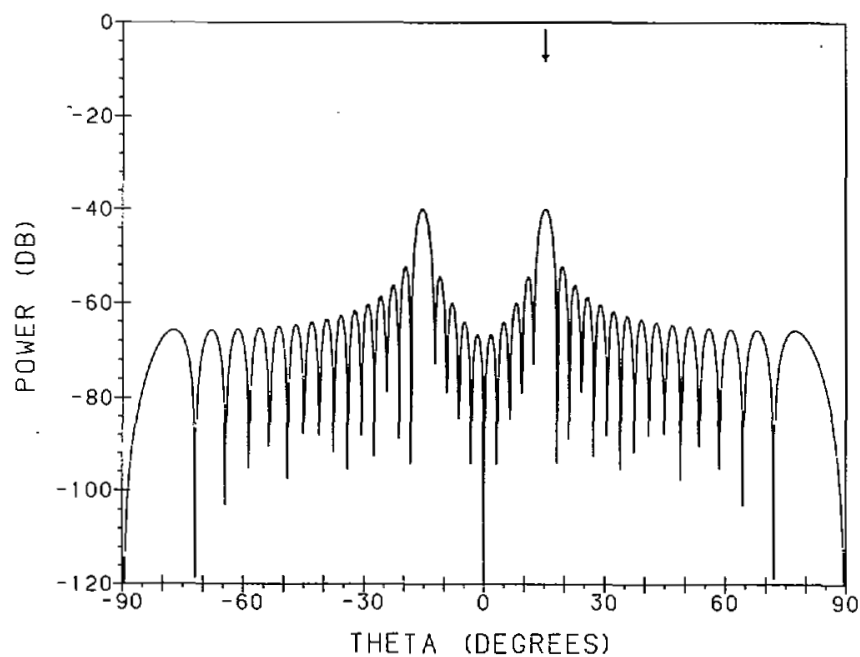

(b)

Fig. 4. (a) Perturbed $40 \mathrm{~dB}$ Chebyshev pattern with one null imposed at $15.23^{\circ}$ with phase-only perturbations. $2 N+1=41, d=\lambda / 2, c_{n}=1$. (b) Cancellation pattern composed of two sinc beams.

However, a general limitation of this approach is that it is incapable of realizing two nulls which are imposed perfectly symmetrically about the main beam. This is a direct consequence of the small angle approximation and the concomitant odd symmetry of the cancellation beams.

\section{B. Large Phase Perturbations}

Large phase perturbations are required when a null is imposed in the main beam vicinity, when two nulls are imposed symmetrically at $u= \pm u_{j}$, when multiple nulls are imposed within a relatively narrow angular sector, or when the number $M$ of imposed nulls increases beyond $M \ll N$. In these cases the phase-only null synthesis problem seems amenable only to numerical solution.

With the nonlinear programming algorithm VMCON [9] we have been successful in obtaining pattern solutions defined by (6). One example of a Chebyshev pattern with two symmetric nulls imposed at $\theta= \pm 14.70^{\circ}$ is shown in Fig. 6. For clarity, only the central portion of the pattern is shown on an expanded scale. Note that the symmetry of the original pattern has been lost in the perturbed pattern. Although the sidelobe level has 


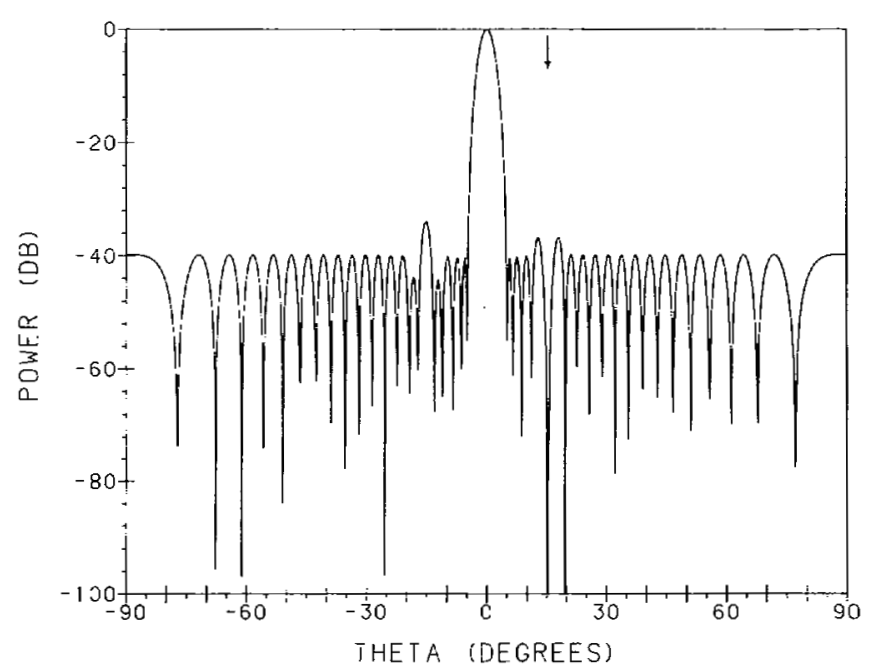

(a)

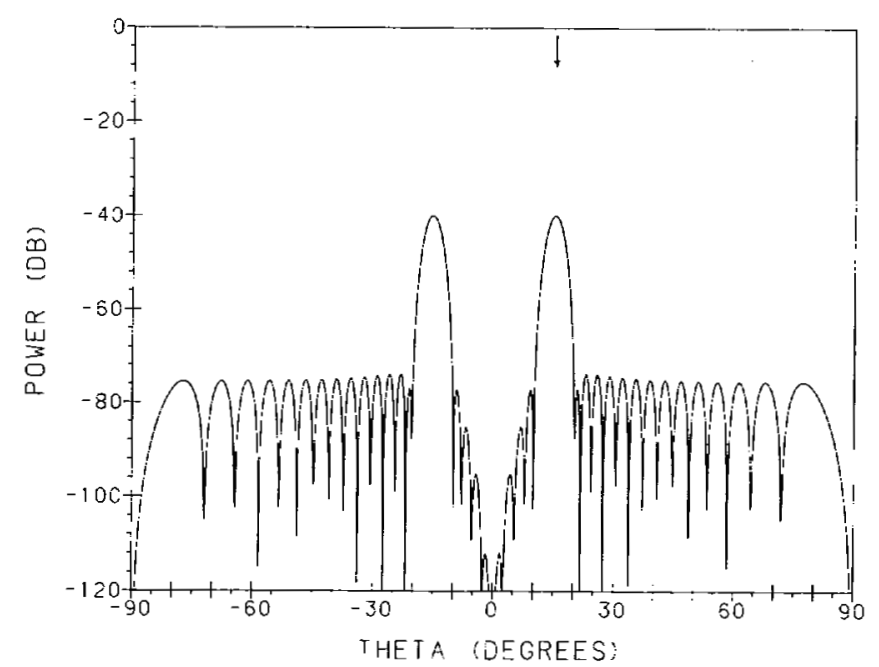

(b)

Fig. 5. (a) Same case as in Fig. 4(a), but $c_{n}=1 / a_{0 n}$. (b) Cancellation pattern composed of two $40 \mathrm{~dB}$ Chebyshev beams.

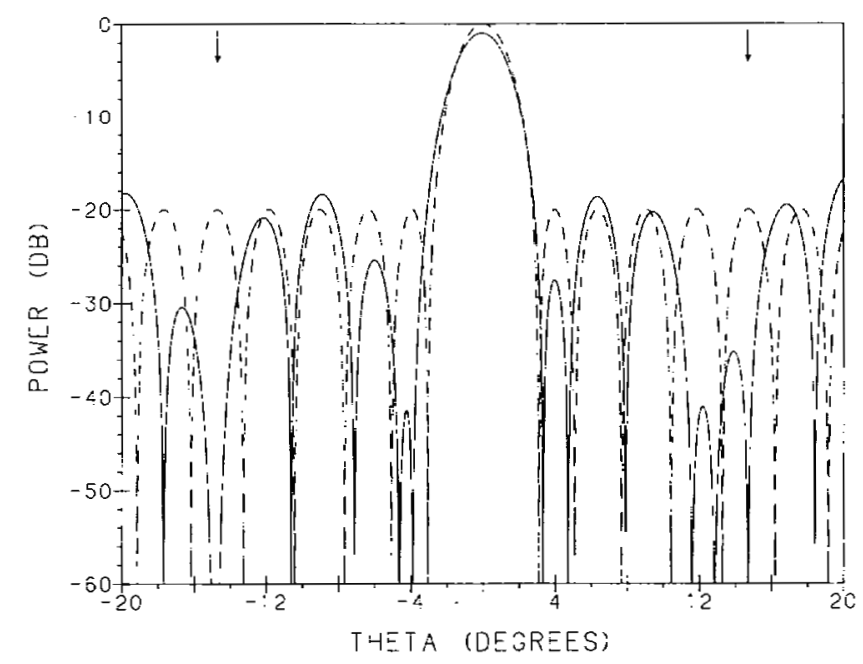

Fig. 6. Original $20 \mathrm{~dB}$ Chebyshev pattern (--) and perturbed pattern ( - ) with nulls imposed at $\pm 14.7^{\circ} .2 N+1=41, d=\lambda / 2, c_{n}=1$. not been increased significantly, the main beam gain has suffered by about $1 \mathrm{~dB}$. Phase perturbations up to $180^{\circ}$ were required. Another example, involving perturbations $\leqslant 110^{\circ}$, is shown in Fig. 7, where four nulls have been symmetrically imposed over the first sidelobe of a sinc-pattern so as to create a wide-band null. As seen this is obtained at the cost of main beam gain and a substantial increase in sidelobe level over the rest of the pattern. The relative merits of searching for a solution in "phase space $\left\{\phi_{n}\right\}$," i.e., solving (6), and in "beamspace $\left\{\gamma_{m}\right\}$," i.e., solving (7), were also investigated [4], by applying these two approaches to the same problem and then comparing the required computation times on a CDC 6600 computer. As seen from Table I, (where the last column corresponds to Fig. 7), beamspace nulling provides a distinct advantage. This is of course to be expected since, in this example, the dimensionality of beamspace ranges only from one to four, whereas it is 41 for phase space.

\section{Nulling with Limited Number of Degrees of Freedom}

Frequently the number of deterministic nulls $M$ is much smaller than the $N-1$ nulls, of which an $N$-element array with full amplitude/phase control is capable. In these situations, null synthesis methods with a reduced number of degrees of freedom become of interest. Two approaches [1] are to implement complex amplitude control at either selected elements in the array aperture or at the subarray level.

The criterion considered in [7] for pattern null synthesis with $Q$ selected elements in the aperture is

$$
\begin{aligned}
& f\left(u_{m}\right) \equiv f_{0}\left(u_{m}\right)+\sum_{q} a_{0 q} \delta_{q} e^{j k d q u_{m}}=0, \quad m=1, \cdots, M \\
& \sum_{q}\left|\delta_{q}\right|^{2}=\text { minimum }
\end{aligned}
$$

where the controllable elements, denoted by $q$, have complex relative amplitude perturbations $\delta_{q}$, such that their total amplitude $a_{q}=a_{0 q}\left(1+\delta_{q}\right)$, and it is assumed that $M<Q$. The corresponding criterion for subarray nulling is

$f\left(u_{m}\right) \equiv f_{0}\left(u_{m}\right)+\sum_{q} \delta_{q} \sum_{n} a_{0 p q} e^{j k d_{p q} u_{m}}=0, \quad m=1, \cdots, M$

$\Sigma\left|\delta_{q}\right|^{2}=$ minimum

where the array has been partioned into $Q$ subarrays with $P$ elements per subarray, with the subscript $p q$ denoting the $p$ th element in the $q$ th subarray and $\delta_{q}$ denoting the weight perturbation of each subarray. The constrained minimization problems defined by (9) and (10) can again be solved analytically.

Fig. 8 shows the result of imposing nulls on the fifth and eighth sidelobe in the pattern of a 20-element array with complex amplitude control at elements 1, 2, 19 and 20. The original pattern, which corresponds to a sampled $30 \mathrm{~dB}$ Taylor distribution, shows surprisingly little perturbation in this case. The location chosen here for the controls at the array edge appears to be optimal [1].

Next, the array was divided into four subarrays, each with 


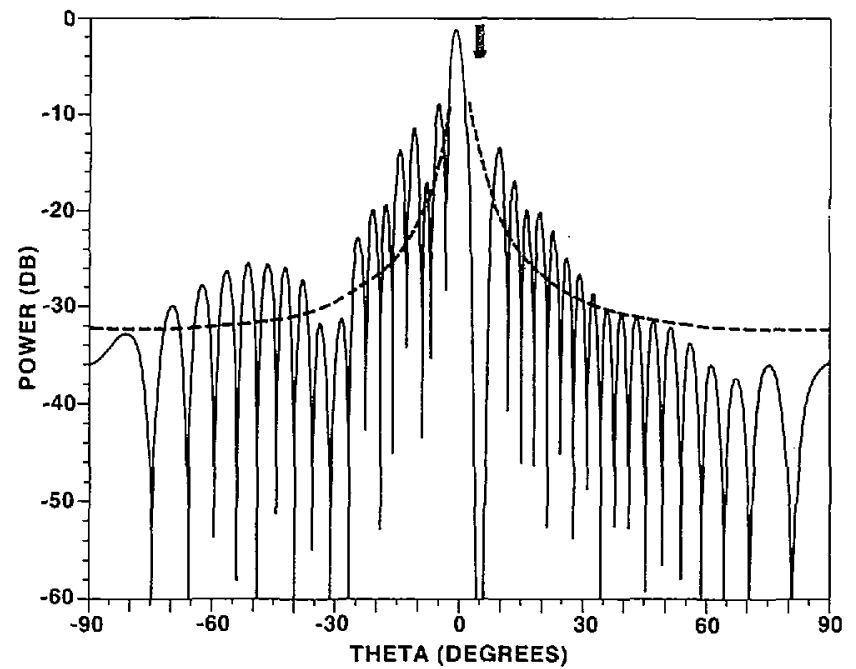

Fig. 7. Envelope of original sinc pattern (--) and perturbed pattern (-) with nulls imposed at $4.0^{\circ}, 4.6^{\circ}, 5.2^{\circ}$ and $5.8^{\circ} .2 N+1=41, d=\lambda / 2, c_{n}=1$.

TABLE I

COMPUTER TIME (SECONDS) FOR BEAMSPACE AND PHASE SPACE PHASE-ONLY NULLING

\begin{tabular}{l|c|c|c|c}
\hline $\begin{array}{l}\text { NUMBER } \\
\text { OF NULLS }\end{array}$ & 1 & 2 & 3 & 4 \\
\hline BEAM SPACE & 0.4 & 0.6 & 0.7 & 2.5 \\
\hline PHASE SPACE & 25.4 & 32.5 & 39.5 & 39.4 \\
\hline
\end{tabular}

Original pattern as in Fig. 7 with nulls imposed at the series of locations $4.0^{\circ}, 4.6^{\circ}, 5.2^{\circ}$ and 5.8 .

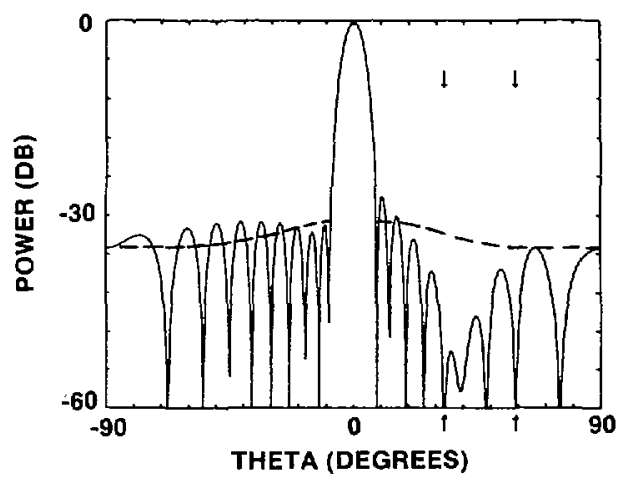

Fig. 8. Original pattern envelope $(-)$ and perturbed pattern (-) with nulls imposed at $33^{\circ}$ and $58^{\circ}$, using four elements with variable weights.

five elements, and the same pattern nulls were imposed at the subarray level. The resultant pattern, shown in Fig. 9(a), is considerably more perturbed than in the previous case. The explanation is that the imposed nulls lie in the sidelobe region of the subarray patterns, whose main beams necessarily are aligned with the original main beam direction. The desired pattern cancellation can therefore be affected only with subarray sidelobes, which leads to subarray weights $\delta_{q}$ of relatively large magnitude. Consequently, in the directions of the main beams and the grating lobes of the subarrays, large perturbations of the original pattern occur.

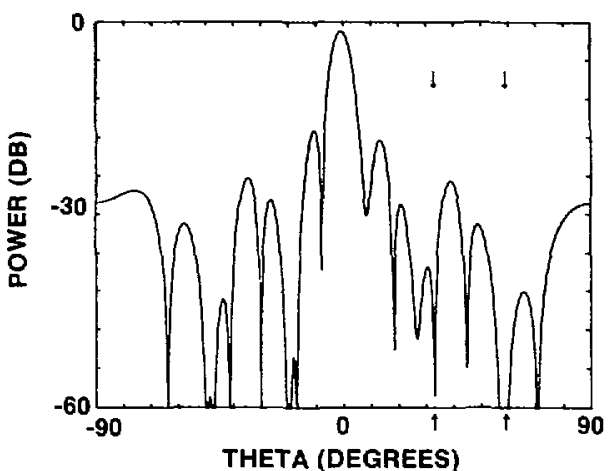

(a)

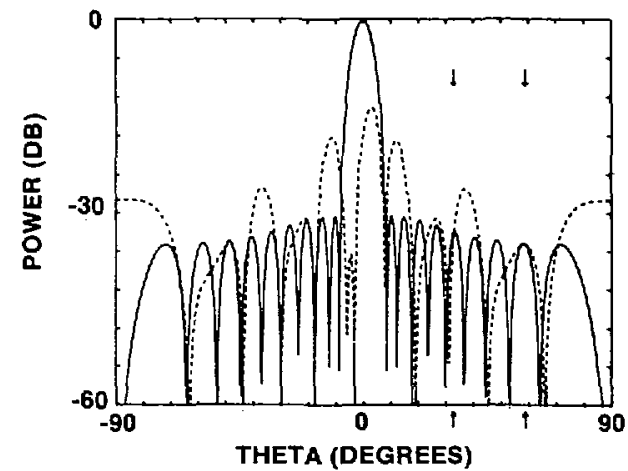

(b)

Fig. 9. (a) Perturbed pattern with nulls imposed at $33^{\circ}$ and $58^{\circ}$ using four subarrays with variable weights. (b) Original pattern (-) and subarray cancellation beam (-).

A conclusion to be drawn [7] is that, for nulls imposed in the sidelobe region, nulling with selected elements in the aperture is the preferred approach. For main beam nulling, on the other hand, this approach will not be efficient since the elements would have to be driven with very large amplitudes. In this case subarray nulling is preferable since each subarray provides a large beam in the main beam direction and therefore these beams can be used efficiently to create a main beam null. A well-known example is a monopulse difference beam, where two subarrays are used to provide the main beam null.

\section{SUMMARY}

We have presented an overview of various pattern null synthesis methods and have discussed their relative merits and limitations. This allows a trade-off between the quality of the constrained pattern and the complexity of electronic control. Obviously, full amplitude/phase control is the superior but also most costly technique. Phase-only control appears to be adequate for most simple nulling situations. The choice between nulling with select array elements or with subarrays depends on whether the nulls are imposed in the sidelobe region or in the main beam region.

The pattern synthesis method, which we have pursued here, is based on constrained mean square approximation. This approach leads to an associated mathematical problem with the reduced dimensionality $M$, the number of imposed nulls only, which is a considerable simplification compared to a problem of dimensionality $2 N+1$, the number of unknown array element excitations. 


\section{REFERENCES}

[1] R. Monzingo and T. Miller, Introduction to Adaptive Arrays. New York: Wiley, 1980.

[2] R. Shore and H. Steyskal, "Nulling in linear array patterns with minimization of weight perturbations," RADC-TR-82-32, (ADA118695), Feb. 1982.

[3] H. Steyskal, "Wide-band nulling performance versus number of pattern constraints for an array antenna." IEEE Trans. Antennas Propagat., vol. AP-31, pp. 159-163, Jan. 1983.

[4] R. Shore, "The use of a beam space representation and nonlinear programming in phase-only nulling," RADC-TR-124, (AD-A131365), May 1983.

[5] _ , "A proof of the odd-symmetry of the phases for minimum weight perturbation, phase-only null synthesis," IEEE Trans. Antennas Propagat., vol. AP-32, pp. 530-533, May 1984.

[6] H. Steyskal, "Simple method for pattern nulling by phase perturbation," IEEE Trans. Antennas Propagat., vol. AP-31, pp. 163-166, Jan. 1983.

[7] R. Haupt, "Nulling with limited degrees of freedom," RADC-TR-83114, (AD-A132276), Apr. 1983.

[8] C. Baird and G. Rassweiler, "Adaptive sidelobe nulling using digitally controlled phase-shifters," IEEE Trans. Antennas Propagat., vol. AP-24, Sept. 1976

[9] R. Crane et al., "Solution of the general nonlinear programming problem with subroutine VMCON," Argonne Nat. Lab., Argonne, IL, Rep. ANL-80-64, 1980.

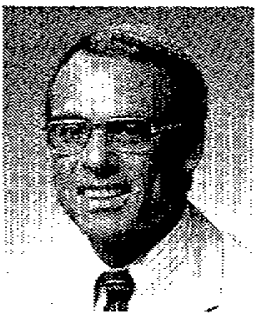

Hans Steyskal (M'76) was born in Berlin, Germany, in 1938. He studied at the Royal Institute of Technology, Stockholm Sweden, from which he received the M.S. degree in 1964 and the Tekn. Dr. degree in 1973.

He joined the Swedish National Defence Research Institute (FOA) in 1962, where he worked on microwave radiation and scattering problems, in particular radar cross section analysis and array antenna pattern synthesis and mutual coupling. During this period he enjoyed two visits with the AF
Cambridge Research Laboratories, on a grant from the Sweden-America Foundation in 1968 and as a Research Associate of the National Research Council in 1973, and also a prolonged "sabbatical" at the Polytechnic Institute of New York in 1976-1978. In 1980 he gave up his position as Chief, Section for Field and Circuit Theory at FOA and moved permanently to the U.S. He now pursues his interests in electromagnetics and applied mathematics at the Electromagnetic Sciences Division of the AF Rome Air Development Center at Hanscom AFB, MA.

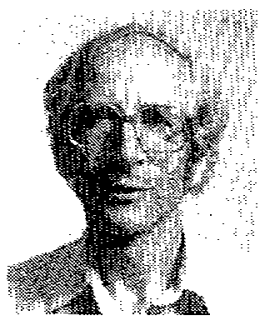

Robert A. Shore was born in Brooklyn, NY. He received the B.A. degree from Amherst College, Amherst, MA, in 1957, and the Ph.D. degree in applied mathematics from Harvard University, Cambridge, MA, in 1968.

He is currently working at RADC in the areas of adaptive arrays and reflector antennas.

Randy L. Haupt (M'82-S'84), for a photograph and biography please see page 493 of the May 1984 issue of this TRANSACTIONS. 